\title{
L-TIROSINA: ANIMALE O VEGETALE?
}

\author{
Nota del m.e. CLAUDIO FUGANTI $(*)$
}

(Adunanza del 18 dicembre 2014)

SuNTO. - Viene presentato uno studio che dimostra come sia possibile avere indicazioni circa la natura animale o vegetale della dieta seguita da un animale mediante la determinazione dell'abbondanza isotopica dell'ossigeno 18 nell'ossidrile fenolico della tirosina, estratta da fonti proteiche provenienti dall'animale stesso.

$* * *$

ABSTRACT. - An investigation has been carried out to show how it is possible to collect information on the animal or vegetal nature of the diet of a certain animal, by determining the $\mathrm{O}^{18}$ isotopic abundance of the $\mathrm{OH}$ group of tyrosine samples extracted from proteins obtained from the animal itself.

Nel 1994 venne il sospetto che il morbo della mucca pazza potesse essere trasmesso fra i ruminanti attraverso la somministrazione, come fonte proteica, di farine di carne e di ossa animali. Nel dicembre 2000 la Commissione Europea vietò l'uso di questi prodotti nella dieta, specie dei vitelli. Da allora, la maggior parte di queste farine animali viene bruciata in centrali termiche e nei cementifici, ma è anche possibile usarle come fertilizzante organico. Allo stesso tempo è stato allestito un sistema efficiente di controllo della presenza dei prioni come agenti induttori della malattia operando sull'animale macellato.

(*) Dipartimento di Chimica del Politecnico di Milano, Italy.

E-mail: mariaelisabetta.brenna@polimi.it 
Tuttavia, non sono disponibili metodi analitici in grado di stabilire se un animale è stato alimentato, in violazione del divieto, con proteine animali. Si ipotizzò che metodi isotopici potessero essere utili allo scopo e lo studio della composizione isotopica dell'ammino acido $L$-tirosina apparve il più indicato. Questo amminoacido aromatico non è essenziale per gli animali che lo possono sintetizzare dall'amminoacido $L$ - fenilalanina per ossidrilazione in posizione para. E' stato altresì dimostrato ${ }^{1}$ che nell'uomo l'intrinseca ossidrilazione della fenilalanina è inversamente proporzionale alla quantità di tirosina presente nella dieta (Fig. 1).<smiles>N[C@@H](Cc1ccc(O)cc1)C(=O)O</smiles>

L-Tirosina<smiles>N[C@@H](Cc1ccccc1)C(=O)O</smiles>

L-Fenilalanina

Fig. 1.

Pertanto, la $L$-tirosina nelle proteine animali deriva in parte dalla dieta e in parte dall'autoproduzione dalla $L$-fenilalanina. In Fig. 2 è riportato lo schema biosintetico di formazione nelle piante della $L$-tirosina e della $L$-fenilalanina. Composto chiave è l'acido arogenico che per decarbossilazione ossidativa dà luogo al primo amminoacido. Quando invece assieme ad una molecola di anidride carbonica viene persa una molecola d'acqua si ha la $L$-fenilalanina. Gli animali sono in grado di ossidrilare la fenilalanina, sintetizzando così la tirosina. Le piante non hanno questa capacità enzimatica, ma sono in grado di ossidrilare in posizione para l'acido cinnamico (con l'ausilio di enzimi del tipo P-450) che, a sua volta deriva dalla $L$-fenilalanina per eliminazione degli elementi dell'ammoniaca, assistita dall'enzima fenilalanina-ammonia-liasi (PAL). E' altresì noto che il sistema enzimatico che negli animali assiste l'ossidrilazione in para della fenilalanina è lo stesso che nelle piante catalizza l'ossidrilazione dell'acido cinnamico. A proposito delle trasformazioni ora nominate è essenziale osservare che l'ossigeno in posizione para della tirosina generata dall'acido arogenico nelle piante deriva, via eritrosio fosfato, dall'acqua delle foglie mentre quello presente nella stessa posizione ed introdotto nella fenilalanina per ossidrilazione diretta proviene dall'ossigeno dell'aria. ${ }^{2}$

La distribuzione degli isotopi stabili di una specie atomica costituente un composto organico non è statistica, ma dipende piuttosto dal modo in cui questo è stato formato. In particolare, l'ossigeno possiede 
gli isotopi $16(99,762 \%), 17(0,038 \%)$ e $18(0.200 \%)$. Il presente studio mostra che la determinazione dell'abbondanza isotopica dell'ossigeno $18\left(\mathrm{O}^{18}\right)$ nell'ossidrile fenolico della tirosina estratta da fonti proteiche definite è utile ai fini dell'identificazione dell'origine animale (autoproduzione) o vegetale dell'amminoacido da cui derivano indicazioni sulla dieta seguita dall'animale.

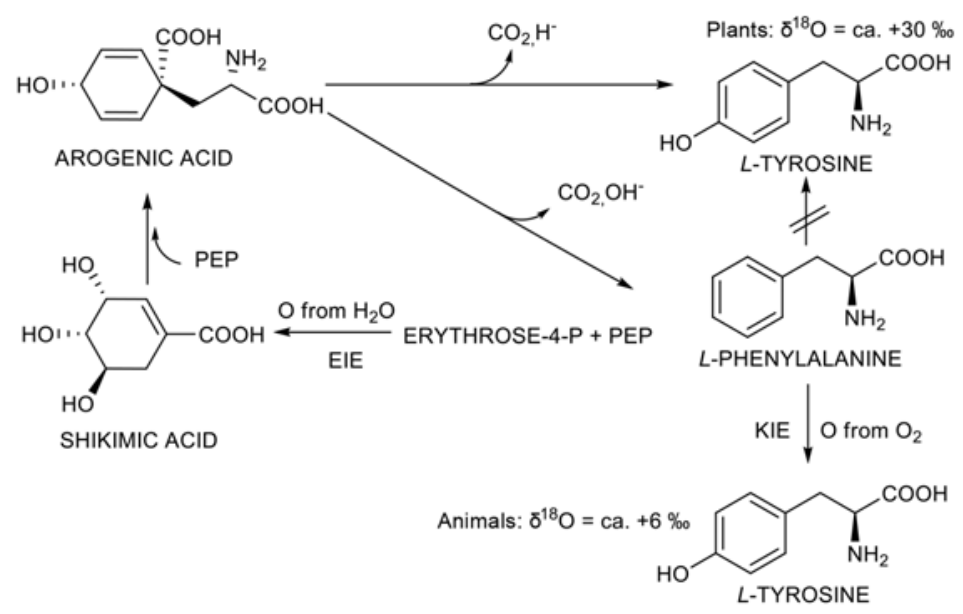

Fig. 2 - Biosintesi della Tirosina.

Le tre fonti primarie di ossigeno per i composti organici (anidride carbonica, ossigeno atmosferico e acqua) posseggono i seguenti arricchimenti in ossigeno $18\left(\delta^{18} \mathrm{O} \%\right.$ ), rispetto all'acqua dell'oceano presa come standard. $\mathrm{CO}_{2}=+40.3 \%$ - $+42.5 \%$; $\mathrm{O}_{2}=+23.5 \%$ - $+23.8 \%$ o e $\mathrm{H}_{2} \mathrm{O}=-10 \%$ - $0 \%$, ove

$$
\delta^{18}(\% \text { o })_{\text {smow }}=\left\{\left[\left({ }^{18} \mathrm{O} /{ }^{16} \mathrm{O}\right)_{\text {campione }} /\left({ }^{18} \mathrm{O} /{ }^{16} \mathrm{O}\right)_{\text {smow }}\right]-1\right\} \times 1000
$$

$$
\text { smow }=\text { standard mean ocean water }
$$

E' stato dimostrato ${ }^{3}$ che nelle piante l'ossidrile in para della tirosina, conservato dall'acido arogenico, deriva dall'acqua delle foglie con valori attesi di $\delta^{18} \mathrm{O}=+27 \%$ - $+30 \%$. Questa circostanza si realizza a causa di un effetto isotopico di equilibrio durante la costruzione dell'eritrosio fosfato nei primi stadi della biosintesi. Invece, l'ossidrile in para della tirosina sintetizzata dalla fenilalanina deriva dall'ossigeno dell'aria, che pos- 
siede $\delta^{18} \mathrm{O}=+23.5 \%$. L'enzima che catalizza l'ossidrilazione della fenilalanina negli animali è lo stesso che assiste la introduzione dell'ossidrile in para dell'acido cinnamico. In questa operazione si verifica un effetto isotopico cinetico. E' stato dimostrato che l'ossigeno in para dell'anello aromatico di composti quali il raspberry ketone e la vanillina, formati nelle piante per ossidrilazione in para dell'anello aromatico dell'acido cinnamico, possiede valori di $\delta^{18} \mathrm{O}$ compresi fra $+5.8 \%$ e $+7 \%$. ${ }^{4}$

Ciò premesso, ci si attende quindi che la tirosina 'vegetale' possegga valori di $\delta^{18} \mathrm{O}$ per l'ossidrile fenolico intorno a $+27 \%$ - + 30\%o mentre quella 'animale' fra $+6 \%$ e $+7 \%$. Nel caso in cui ci sia stato da parte dell'animale assunzione di tirosina prevalentemente dalla dieta vegetale, con poca o nessuna autoproduzione dalla fenilalanina, si attendono valori elevati di $\delta^{18} \mathrm{O}$. Questi valori scenderanno se l'animale si è 'autoprodotto' l'amminoacido per ossidrilazione della fenilalanina con ossigeno atmosferico.

Per verificare questa ipotesi si è degradata la tirosina a dare 3-(4metossifenil)-1-cloropropano ( $p-\mathrm{CH}_{3} \mathrm{OC}_{6} \mathrm{H}_{4} \mathrm{CH}_{2} \mathrm{CH}_{2} \mathrm{CH}_{2} \mathrm{Cl}$ ), che ritiene come unico ossigeno quello in posizione para (Fig. 3) e si sono determinati i valori di $\delta^{18} \mathrm{O}$ di un set di materiali identici derivati da campioni di tirosina di differenti origini. I risultati sono elencati in Tab. 1. Questa comprende anche i valori di $\delta^{18} \mathrm{O}$ relativi all'ossigeno in para della vanillina (che ci si attende sia lo stesso della tirosina completamente 'animale' ottenuta in vivo dalla fenilalanina) e quelli del derivato monoossigenato ottenuto dal tirosolo $\left(p-\mathrm{HOC}_{6} \mathrm{H}_{4} \mathrm{CH}_{2} \mathrm{CH}_{2} \mathrm{OH}\right)$, metabolita della tirosina isolato dal Ligustrum ovalifolium raccolto nelle vicinanze di Brescia (corrispondente al valore di una tirosina totalmente 'vegetale').
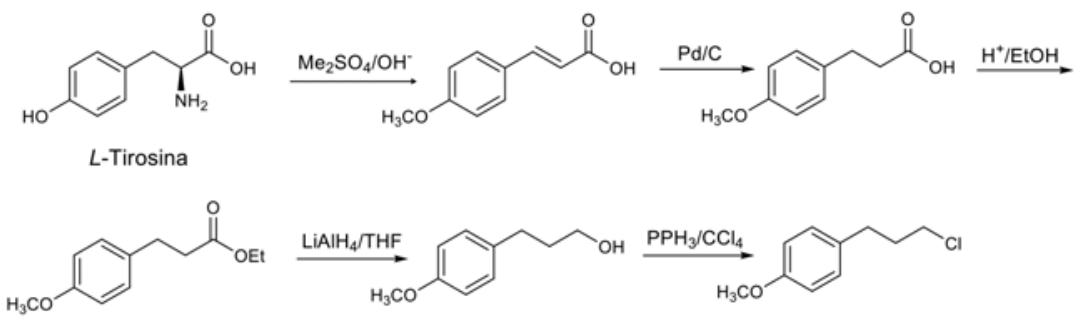

Fig. 3 - Degradazione della tirosina a composto mono ossigenato.

Fra i campioni commerciali di tirosina (3-7) di origine non definita si osservano valori compresi fra $+19.6 \%$ e $+27.5 \%$ - + $31.5 \%$ o campioni 7 e 3). Questi numeri sono simili come grandezza a quelli del tirosolo dal vegetale Ligustrum ovalifolium (+26.2\%). Questo ultimo valo- 
re sperimentale dovrebbe essere corretto a $+30.2 \%$ se si ipotizza che il valore di $\delta^{18} \mathrm{O}$ dell'acqua del suolo della zona di Brescia sia analogo a quello di Milano (ca. - 7\%o). Molto interessanti, tuttavia, appaiono i dati relativi ai tre campioni di tirosina 8,9 e 10 . In particolare, i valori scendono dal primo campione (n 8) (caseina vaccina $\delta^{18} \mathrm{O}=+23.5 \%$ ) al terzo (n 10) (capelli umani $\delta^{18}==+17.8 \%$ ) passando per il terzo (piume di gallina ${ }^{18} \mathrm{O}=+19.8 \%$ ). I valori quindi scendono da quello del tirosolo e si avvicinano a quello della vanillina (rappresentativo di una tirosina idealmente tutta 'animale').

Tab. 1 - Origine dei composti aromatici e valori di $\delta^{18} \mathrm{O}$ dell' $\mathrm{OH}$ fenolico in posizione para.

\begin{tabular}{lcc}
\hline COMPOSTO & ORIGINE & $\delta^{18} \mathbf{O}$ \\
\hline 1. Vanillina & Vanilla planifolia & +5.8 \\
\hline 2. Acido gallico & Rhus sp. & +29.8 \\
\hline 3. Tirosina & Commerciale, Aldrich & +31.5 \\
\hline 4. L-Tirosina & Commerciale, Fluka & +22.7 \\
\hline 5. L-Tirosina & Commerciale, Merck & +23.7 \\
\hline 6. L-Tirosina & Commerciale, ignota & +19.6 \\
\hline 7. D,L-Tirosina & Commerciale, Fluka & +27.5 \\
\hline 8. L-Tirosina & Da idrolisi caseina & +23.5 \\
\hline 9. L-Tirosina & Da piume di gallina & +18.9 \\
\hline $\mathbf{1 0 .}$ L-Tirosina & Da capelli umani & +17.8 \\
\hline $\mathbf{1 1 .}$ Tirosolo & Sintetico, Janssen & +18.6 \\
\hline $\mathbf{1 2 .}$ Tirosolo & Ligustrum ovalifolium & +26.2 \\
\hline $\mathbf{1 3 .}$ 2,3,5-Trimetil fenolo & Sintetico, Merck & +9.5
\end{tabular}

Nella Fig. 4 sono correlati i valori di $\delta^{18} \mathrm{O}$ dei campioni di origine nota 8 , 9 e 10 con i valori $+6 \%$ e, rispettivamente, $+30 \%$ o corrispondenti alle due tirosine completamente 'animali' e, rispettivamente, 'vegetali'. In questo modo appare che la tirosina di origine 'vegetale' sia presente per il $73 \%$ nella caseina, per il $54 \%$ nelle piume di gallina e, infine, per il $49 \%$ nei capelli umani.

Il lavoro di caratterizzazione isotopica della tirosina e dei prodotti correlati sopra riportato è stato successivamente sviluppato dal prof. H.-L- Schmidt e collaboratori che hanno caratterizzato dal punto di vista isotopico la tirosina attraverso due modi di degradazione a prodotti che contengono solo l'ossidrile fenolico, basati su degradazioni enzimatiche sofisticate. In un caso, usando l'enzima che degrada la tiro- 
sina a fenolo, piruvato e ammoniaca si è ottenuto il fenolo, analizzato come tribromo derivato.

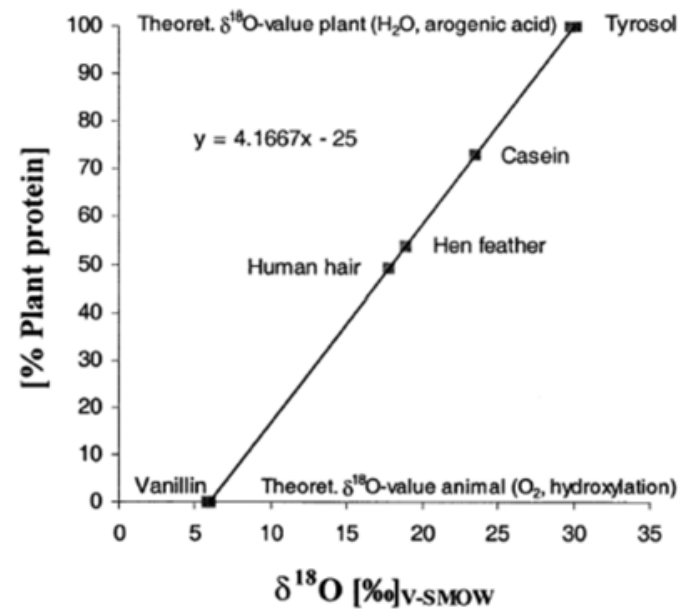

Fig. 4.

Alternativamente, si è trasformata la tirosina mediante l'uso della tirosina decarbossilasi in tiramina, analizzata come tale. I valori di $\delta^{18} \mathrm{O}$ ottenuti su un set di campioni molto variegato (Tab. 2) e riportati nella Fig. 5 permettono, comparando i valori di $\delta^{18} \mathrm{O}$ di tirosina dai muscoli di carnivori quali il coccodrillo, il cane e il gatto, con quelli di erbivori di concludere che se i valori di $\delta^{18} \mathrm{O}$ sono inferiori a $+19.5 \%$ o c'è alta probabilità che l'animale sia stato alimentato come un 'carnivoro'. ${ }^{6}$

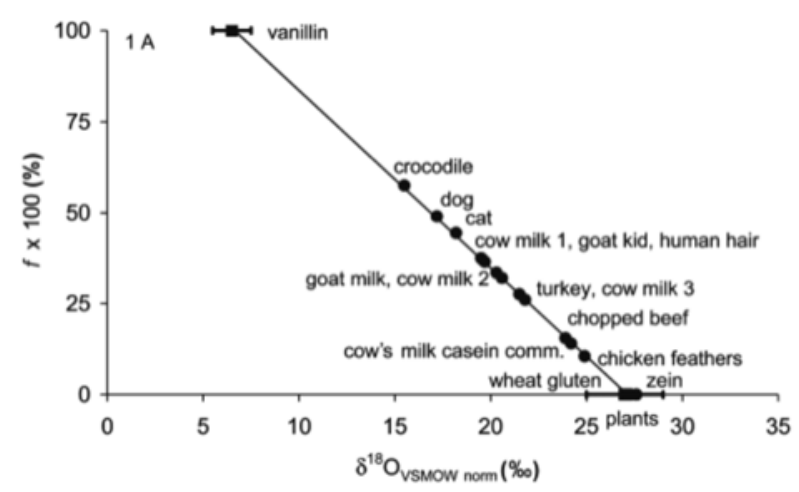

Fig. 5 . 
Tab. 2 - Valori di $\delta^{18} \mathrm{O}$ dell'ossidrile fenolico della tirosina proteica di diverse origini.

\begin{tabular}{lccc}
\hline Proteina & Storia dietetica & $\mathbf{\delta}^{18} \mathbf{O}$ & $\mathbf{F} \mathbf{x} \mathbf{1 0 0}$ \\
\hline Glutine di grano & & +27.3 & \\
\hline Zeina & & +27.6 & \\
\hline Carne macinata & Sconosciuta & +23.9 & 15.5 \\
\hline Caseina commerc. & Sconosciuta & +24.2 & 14.0 \\
\hline Caseina 1 (mucca) & Erba & +19.2 & 37.5 \\
\hline Caseina 2 (mucca) & Erba, fieno & +20.6 & 32.0 \\
\hline Caseina 3 (mucca) & Grano, insilato & +21.8 & 26.0 \\
\hline Caseina (capra) & Erba, fieno & +20.3 & 33.5 \\
\hline Capretto (muscolo) & Solo latte di capra & +19.7 & 36.5 \\
\hline Tacchino (muscolo) & Sconosciuta & +21.5 & 27.5 \\
\hline Piume di gallina & Sconosciuta & +24.9 & 10.5 \\
\hline Capelli umani & Sconosciuta & +19.6 & 27.0 \\
\hline Cane (muscolo) & Sconosciuta & +17.2 & 49.0 \\
\hline Gatto (muscolo) & Sconosciuta & +18.2 & 44.4 \\
\hline Coccodrillo (muscolo) & Carne di manzo & +15.5 & 57.5 \\
\hline
\end{tabular}

$\left[\mathrm{f}=\left(27-\delta^{18} \mathrm{O}_{\text {norm }}\right) / 20\right]$.

\section{BIBLIOGRAFIA}

1. Thorpe, J.M., Roberts, S.A., Ball, R.O., Pencharz, P.B. Metabolism, 2000, 49, 444-449.

2. Haslam, E. Shikimic Acid, metabolism and metabolites Wyley \& Sons, Chichester 1993.

3. Schmidt, H.-L., Werner, Rossman, A. Phytochem., 2001, 58, 9-32.

4. Fronza, G., Fuganti, C., Pedrocchi-Fantoni, G., Serra, S., Zucchi, G., Fauhl, C., Guillou, C., Reniero, F. J. Agric. Food Chem., 1999, 47, 1150-1155.

5. Fronza, G., Fuganti, C., Schmidt, H.-L, Werner, R. Eur. Food Res. Technol., 2002, 215, 55-58.

6. Tanz, N., Werner, R., Eisenreich, W., Schmidt, H.-L. J. Agric. Food Chem., 2011, 59, 9475-9483. 